\title{
Fabrication and Simulation of Self-Focusing Field Emission X-ray Tubes
}

\section{Kevin Cheng ${ }^{1}$, Yi-Ping Chou ${ }^{1}$, Meng-Jey Youh ${ }^{2, \dagger}$, Yih-Ming Liu ${ }^{3, *}$, Ming-Der Ger ${ }^{3, \dagger}$, Kung-Hsu Hou ${ }^{4, \dagger}$ and Nen-Wen Pu ${ }^{5, *}$}

1 School of Defense Science, Chung Cheng Institute of Technology, National Defense University, Dasi, Taoyuan 335, Taiwan; E-Mails: kevin.cheng.ndu@gmail.com (K.C.); koleon2001@yahoo.com.tw (Y.-P.C.)

2 Department of Information Technology, Hsing Wu University, New Taipei City 244, Taiwan; E-Mail:mjyouh@gmail.com

3 Department of Chemical and Materials Engineering, Chung Cheng Institute of Technology, National Defense University, Dasi, Taoyuan 335, Taiwan; E-Mail: mingderger@gmail.com

4 Department of Power Vehicle and Systems Engineering, Chung Cheng Institute of Technology, National Defense University, Dasi, Taoyuan 335, Taiwan; E-Mail: khou@ndu.edu.tw

5 Department of Photonics Engineering, Yuan Ze University, Chung-Li, Taoyuan 320, Taiwan

$\dagger$ These authors contributed equally to this work.

* Authors to whom correspondence should be addressed; E-Mails: liuym@ndu.edu.tw (Y.-M.L.); nwpuccit@saturn.yzu.edu.tw (N.-W.P.); Tel.: +886-3-380-1126 (ext. 316526) (Y.-M.L.); +886-3-463-8800 (ext. 7512) (N.-W.P.); Fax: +886-3-389-2494 (Y.-M.L.); $+886-3-451-4281$ (N.-W.P.).

Academic Editor: Wen-Hsiang Hsieh

Received: 27 July 2015 / Accepted: 20 October 2015 / Published: 26 October 2015

\begin{abstract}
A self-focusing field emission (FE) X-ray tube with a large-area cathode design was simulated and fabricated. The designed X-ray tube had a cylindrically symmetric geometry; the diameter of the cathode and the anode was $15 \mathrm{~mm}$, and the cathode-anode distance was $20 \mathrm{~mm}$. Owing to the unique cup-shaped design of the cathode, the electron beam emitted from the large-area cathode was focused onto the anode without using magnetic lenses or extra biased electrodes. Carbon nanocoils, which were grown on the bottom of the circular cup-shaped cathode, were used as electron emitters because of their excellent FE properties. A simulation of the electron trajectories for various cup heights revealed that the optimal focal spot size $(0.1 \mathrm{~mm})$ was obtained at a cup height of $5 \mathrm{~mm}$
\end{abstract}


when a voltage of $50 \mathrm{kV}$ was applied. To verify this result, an X-ray tube was fabricated and tested. An X-ray photograph of the tested tooth and electric circuits showed good resolution and X-ray intensity. The large cathode area effectively reduces the current density and thereby improves the lifetime of the cathode.

Keywords: X-ray tubes; field emission; carbon nanocoils

\section{Introduction}

X-ray radiation has long been used in various fields, such as medical diagnosis, industrial applications, material characterization, and scientific research. A traditional X-ray tube comprises a metal filament (hot cathode) and a metal target (anode), which are enclosed in an evacuated glass envelope. The metal filament is heated to over $1000{ }^{\circ} \mathrm{C}$ to emit electrons, which are accelerated by an applied electric field to bombard the metal target, generating X-rays [1]. These thermionic emission (TE) X-ray tubes have many limitations. Metal filaments must be heated to a high temperature to boil off electrons, so the response time is long. Moreover, the energy distribution of the emitted electrons from the heated cathode is wide, inhibiting their focusing and the X-ray imaging resolution. Also, the high temperature may lead to evaporation of the filament material or its oxidation by residual gases, reducing its lifetime. The typical lifetime of a TE X-ray tube is less than a year and a large portion of their failures thereof is filament-related.

Field emission (FE) of electrons from the surface of a conductor was proposed by Fowler and Nordheim in 1928 [2]. In FE, electrons are drawn from high-aspect-ratio or low-work-function cathode materials to the vacuum under a high electric field. This phenomenon is used in a variety of applications such as FE display panels [3], backlight units [4], field emission lamps (FELs) [5], and $\mathrm{X}$-ray tubes [6]. FE-type X-ray tubes have many desirable properties. Electrons are extracted from the metal cathode by an applied electric field while the temperature of the emitters is significantly lower than that of the TE filaments. A colder cathode has the benefits of a longer lifetime and a much faster response. The FE current depends on the applied voltage, and the X-ray intensity depends on the FE current. Thus, the X-ray intensity can be conveniently and very rapidly controlled by varying the cathode voltage. Many researchers have worked on the design and fabrication of FE X-ray tubes [7,8].

The typical FE X-ray tubes may comprise three parts, which are an FE cathode grown with electron emitters, an anode (metal target), and a gate electrode. Also, magnetic lenses or extra biased focusing electrodes can be used to focus the electrons onto the anode. Since nano-materials were discovered, carbon nanotubes (CNTs) [9-11], carbon nano-coils (CNCs) [12-15], and CNTs decorated with nanodiamonds [16] have been identified as excellent FE cathode materials because of their high aspect ratio, good thermal stability, and good mechanical strength. The extremely high aspect ratios of CNTs and $\mathrm{CNCs}$ give rise to a large field enhancement factor, $\beta$ [17], which makes them excellent field emitters with low turn-on fields. Researchers have found that CNCs exhibit several advantages over CNTs, including better long-term current emission stability, higher current density, and longer operation lifetime $[18,19]$. 
The resolution of an X-ray image depends on the spot size of the electron beam on the anode. To minimize the spot size, a focusing electrode [20] is commonly used, but its use requires an extra bias circuit and results in a more complicated tube design. In this research, a simple diode-type FE $\mathrm{X}$-ray tube is designed and focusing is achieved without resorting to magnetic lenses or active focusing electrodes.

\section{Simulation Methodology, Material, and the Experiments}

This section will firstly introduce the simulation methodology, and then describe the material properties of CNCs and the process of their fabrication.

\subsection{Finite Element Method (FEM)}

The FEM was employed in the simulation to calculate the electric field strength. The FEM is a numerical technique for finding approximate solutions to boundary value problems for differential equations. First, the problem domain is divided into finite elements, as shown in Figure 1, and each element is represented by a set of element equations to the original problem. To make the final calculation, all element equations are systematically recombined into a global system of equations, which can be numerically solved by known techniques from the initial values in the original problem.

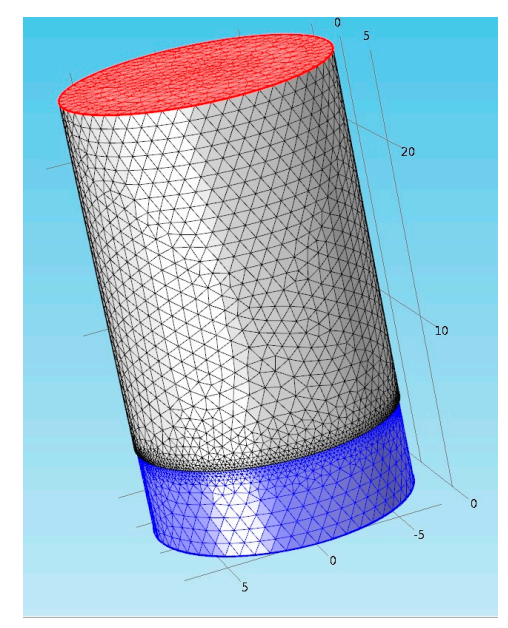

Figure 1. X-ray tube divided into finite elements for electric field calculations. Red and blue parts are anode and cathode, respectively.

The FEM is the preferred method in structural mechanical analysis. In this study, the FEM is a suitable tool for obtaining a numerical solution for the spatial distribution of the electric field between the cathode and the anode.

\subsection{Field Emission Theory}

FE refers to the quantum-mechanical phenomenon of electron tunneling from a conductor into the vacuum through a surface potential barrier whose width is significantly reduced by a high local electric field [21-25]. The tunneling probability depends very strongly on the width of the potential barrier and decreases exponentially with increasing barrier width. The local electric field can be orders 
of magnitude greater than the applied macroscopic field, owing to the enhancement effect at sharp tips or edges.

The FE current $(I)$ can be calculated from the quantum-mechanical tunneling probability of electrons through a triangular potential barrier, and the result is the well-known Fowler-Nordheim (F-N) equation:

$$
I=\alpha A\left(\beta^{2} E^{2} / \phi\right) \exp \left(-b \phi^{3 / 2} / \beta E\right)
$$

where $\alpha$ and $b$ are constants; $A$ is the effective FE area; $E$, $\phi$, and $\beta$ are the applied macroscopic electric field (without the local microscopic geometrical effect that causes enhancement), the material work function, and the field enhancement factor, respectively. The value of $\beta$ depends on the microscopic geometry of the emitters and is the most important factor that affects the FE performance. Nanomaterials with enormously high aspect ratios, such as CNCs and CNTs, are known to have very high $\beta$, and have been demonstrated to be excellent field emitters with low turn-on $E$ fields [21-25]. The value of $\beta$ can be extracted from the experimentally obtained slope of the F-N plot.

To obtain the macroscopic electric field $E$, which determines the FE current density, Poisson's equation was firstly solved numerically for the potential distribution $V(x, y, z)$ using the FEM. The electric field distribution is then calculated by differentiation:

$$
\vec{E}=-\vec{\nabla} V
$$

In this work, three different kinds of software packages were used in the simulation; they were (1) SolidWorks ${ }^{\circledR}$ (Dassault Systèmes SolidWorks Corp., Waltham, MA, USA), which was used to define a precise device geometry; (2) Comsol Multiphysics (Comsol Inc., Stockholm, Sweden), which was used to calculate the distribution of the electric field on the designed cathode surface; and (3) Simion 8.0 (Scientific Instrument Services, Inc., Ringoes, NJ, USA), which simulated the trajectory of the emitted electrons that were accelerated by the electric field between the cathode and the anode.

\subsection{CNCs as Electron Emitting Nanomaterial}

The unique properties of carbon nanomaterials, such as their high thermal conductivity, low work function, and excellent electron migration rate, make them promising FE emitters. CNTs have received much attention since their discovery by Iijima [26-32]. An advantage of CNTs in FE is that they are natural field-enhancing objects because of their high aspect ratio.

CNCs [13-16,33-37] are nanocarbon materials with a coiled structure and are composed of nanometer-scale fibers. Compared with CNTs, CNCs have considerably more electron emission sites - not only at the tips but also at the numerous nanocrystallites embedded in the amorphous carbon body. Additionally, CNCs are more robust than CNTs because the former are much thicker and thus can withstand a higher current density. Owing to their much larger diameter and higher FE site density, CNCs exhibit better long-term current emission stability, current density, FE efficiency, and lifetime than CNTs [18,19], and so they have already found application in FE lighting [38-41]. Consequently, CNCs are used as the emitter material in the X-ray tube in this work.

CNCs are generally synthesized by catalytic thermal chemical vapor deposition (TCVD) or arc discharge. Since CVD can be used in the low-cost, large-scale commercial synthesis of CNCs, it was utilized to synthesize the CNCs herein. 
The substrates that were used herein for the synthesis of CNC cathodes were 304 stainless steel circular disks with a diameter of $15 \mathrm{~mm}$. Each substrate was firstly cleaned with acetone, ultrasonicated for $10 \mathrm{~min}$, and subsequently washed in deionized water. To expose the bare metal surface, each stainless steel substrate was then immersed in $10 \%-37 \% \mathrm{HCl}$ for $3-10$ min and rinsed again with deionized water. Before TCVD, Pd catalysts were deposited onto the substrates through chemical displacement in a $100-800 \mathrm{ppm} \mathrm{PdCl}_{2}$ solution of $\mathrm{pH} 1.6-1.8$ at $80{ }^{\circ} \mathrm{C}$ for $10 \mathrm{~min}$. After the reaction was complete, the cathodes were rinsed with deionized water and dried with $\mathrm{N}_{2}$ gas. The prepared cathodes were placed in a TCVD furnace and the pressure was pumped down to $10^{-2}$ Torr. The temperature was gradually increased to $600{ }^{\circ} \mathrm{C}$ at a rate of $20{ }^{\circ} \mathrm{C} / \mathrm{min}$ in an atmosphere of $\mathrm{H}_{2}$ and Ar. The flow rates for $\mathrm{H}_{2}$ and Ar were 10 and $100 \mathrm{sccm}$, respectively. When the temperature reached $600{ }^{\circ} \mathrm{C}$, the Ar gas was evacuated and the $\mathrm{H}_{2}$ flow rate was increased to $20 \mathrm{sccm}$ to pretreat the $\mathrm{Pd}$ catalysts for $5 \mathrm{~min}$. Next, to grow the $\mathrm{CNCs}, \mathrm{C}_{2} \mathrm{H}_{2}$ gas was fed at a flow rate of $5 \mathrm{sccm}$ for $20 \mathrm{~min}$ while the furnace temperature was maintained at $600^{\circ} \mathrm{C}$.

The morphologies of the CNCs were examined using a transmission electron microscope (TEM, model: Tecnai F30, FEI, Hillsboro, OR, USA) and a field emission scanning electron microscope (FE-SEM, model: JEOL JSM-7600F, Japan Electron Optic Laboratory Co., Ltd., Tokyo, Japan). Their graphite crystallinity was analyzed using Raman spectroscopy.

\section{Results and Discussion}

\subsection{Simulation of the Self-Focusing Cathode Design}

Figure 2 displays the geometry of the X-ray tube for simulation. The red part is the anode, which has a diameter of $15 \mathrm{~mm}$ and is biased at an applied voltage of $50 \mathrm{kV}$; the blue part is the metallic cup for electrostatic focusing which has a diameter of $15 \mathrm{~mm}$ with a 12-mm-diameter aperture on the top to focus the emitted electrons. The CNC FE cathode disk (not shown) was situated at the bottom of the metallic cup. The whole cathode (CNC FE disk and cup) was grounded. The distance from the electron emitters to the anode was fixed at $20 \mathrm{~mm}$, and the cup height was varied to minimize the size of the focal spot on the anode.

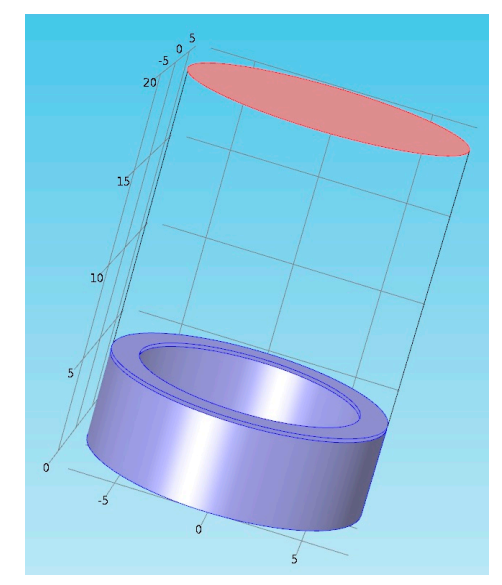

Figure 2. Geometry of the X-ray tube in SolidWorks. Red and blue parts are anode and cup-shaped cathode, respectively. CNC FE disk (diameter $=15 \mathrm{~mm}$ ) is at the bottom offocusing cup; a 12-mm-diameter aperture is at the top of cup. 
In the design herein, the electron-emitting area was quite large. This was advantageous because it would greatly reduce the average current density if the total FE current was fixed, resulting in a longer lifetime of the CNC emitters.

The electrostatic potential distribution and, hence, the electric field distribution were then calculated using Comsol Multiphysics. Finally, the trajectories of the emitted electrons accelerated by the Coulomb force were simulated using Simion 8.0. The calculated trajectories are shown in Figure 3.

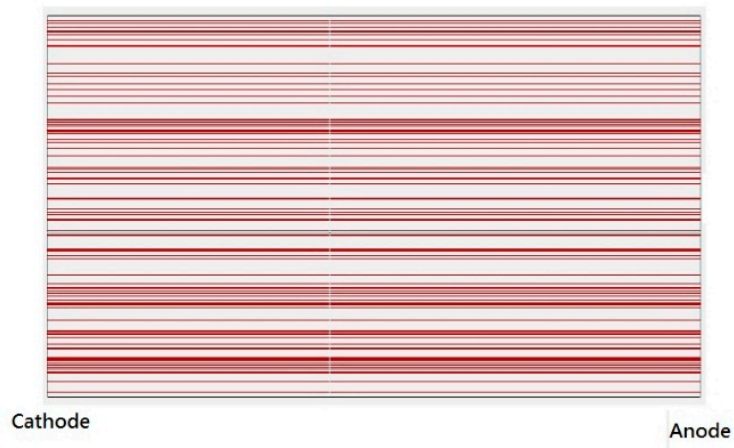

(a)

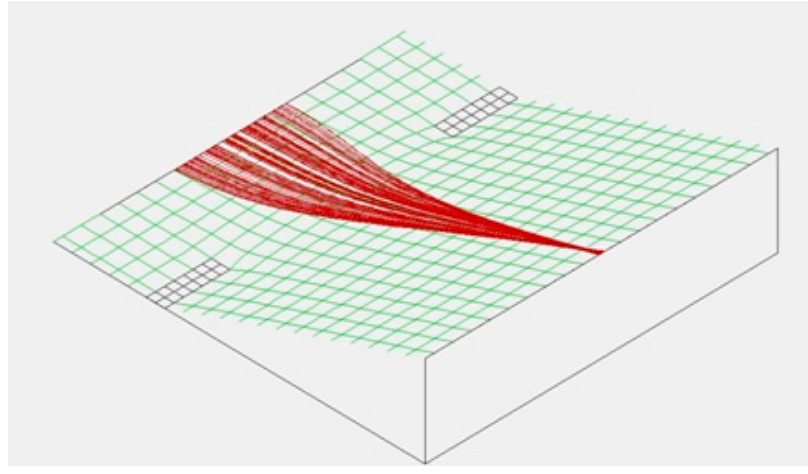

(b)

Figure 3. Trajectories of emitted electrons (a) without and (b) with focusing cup design. Vertical axis in (b) represents electric potential $V$.

Figure 3a presents the simulation results for an X-ray tube without the focusing cup; electrons are dragged by the applied voltage in straight trajectories to bombard the anode. In contrast, in Figure $3 \mathrm{~b}$, the trajectories of the electrons emitted from a cup-shaped cathode clearly converged onto a small focal spot. After testing with various heights of the cup-shaped cathode, the optimal cup height was about $5 \mathrm{~mm}$, which yielded the smallest focal spot size of $0.1 \mathrm{~mm}$ (diameter). Figure 4 shows the three-dimensional (3D) electron trajectories in the X-ray tube with a 5-mm-high focusing cup.

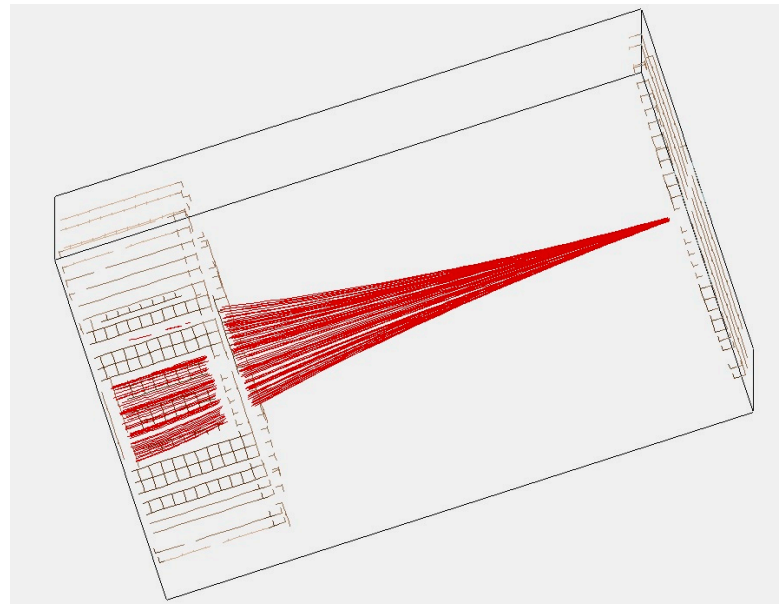

Figure 4. Three-dimensional (3D) diagram of electron trajectories in the X-ray tube with a 5-mm-high focusing cup.

Note that the focusing cup is maintained at the same voltage as the CNC FE disk. Thus, no extra bias circuit is needed, and the structure and fabrication of such an X-ray tube are simple. 
To understand why this simple design offered such a good focusing effect for a large-area cathode, Figure 5 shows the electric field distribution on the CNC FE disk (a) without and (b) with the focusing cup design. Comparing Figure 5a with $\mathrm{b}$, the metallic cup wall clearly has a strong shielding effect: the electric field strength near the disk edge was reduced from $9.5 \times 10^{5} \mathrm{~V} / \mathrm{m}$ (center) to below $1.2 \times 10^{5} \mathrm{~V} / \mathrm{m}$. In contrast, the electric field without the focusing cup was nearly constant everywhere on the CNC FE disk. This shielding effect forced the electric field lines within the cup to converge towards the central axis, so the electrons were guided by this field distribution and focused onto the anode.

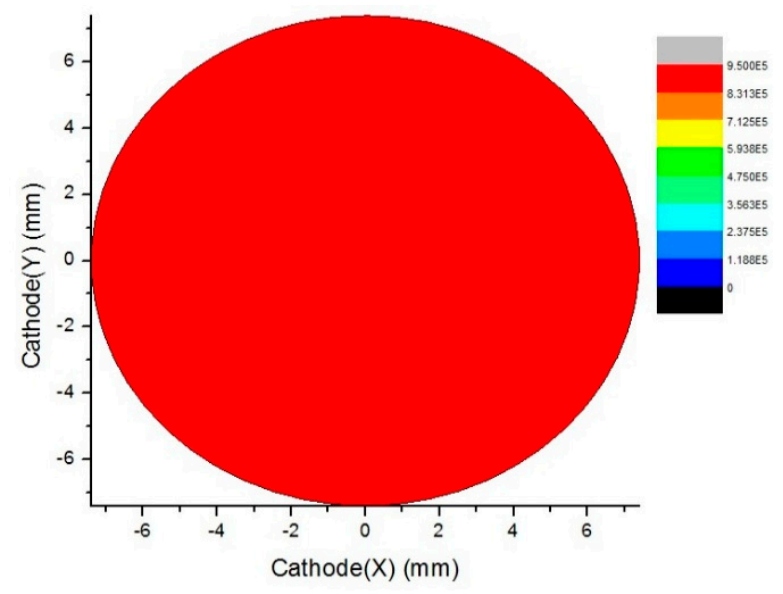

(a)

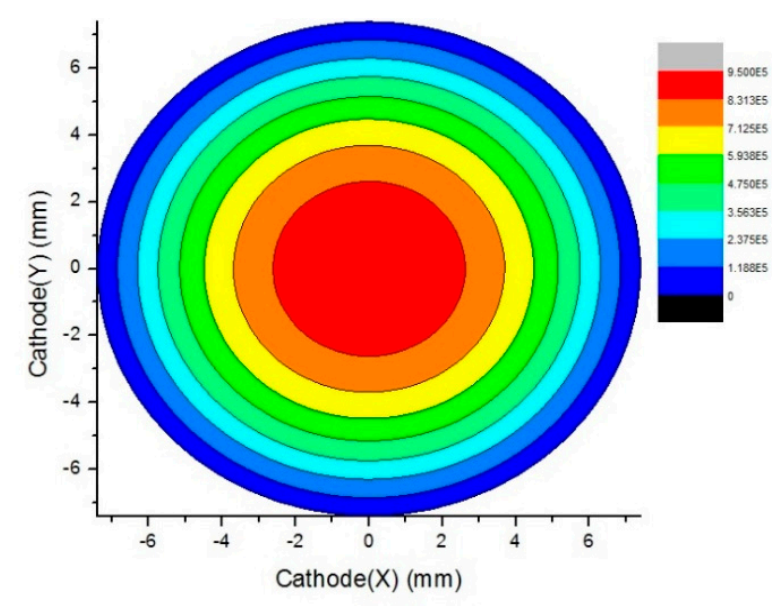

(b)

Figure 5. Calculated electric field strength on the surface of CNC FE disk (a) without and (b) with focusing cup design.

\subsection{Characterization of the Synthesized CNCs}

Figure 6 (secondary electron image) displays a scanning electron micrograph (SEM) of the CNCs grown on the stainless steel disk. The pitch and diameter of the CNCs are both approximately $0.1-0.2 \mu \mathrm{m}$.

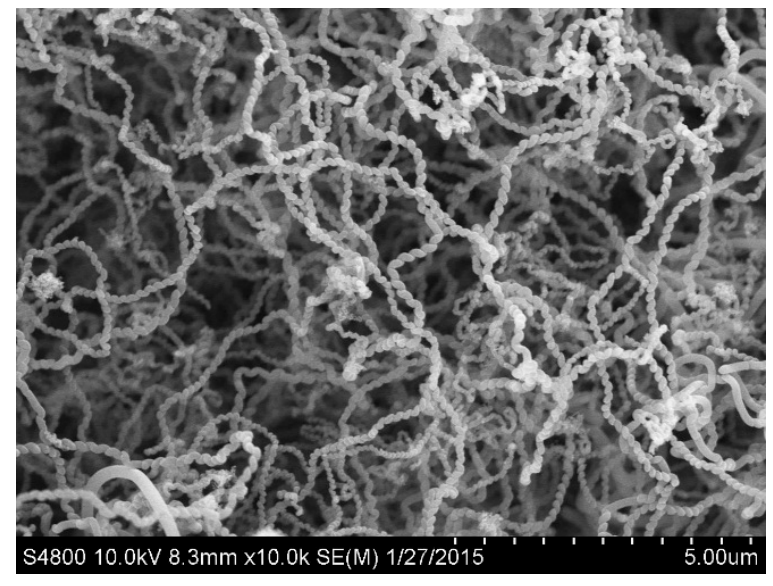

(a)

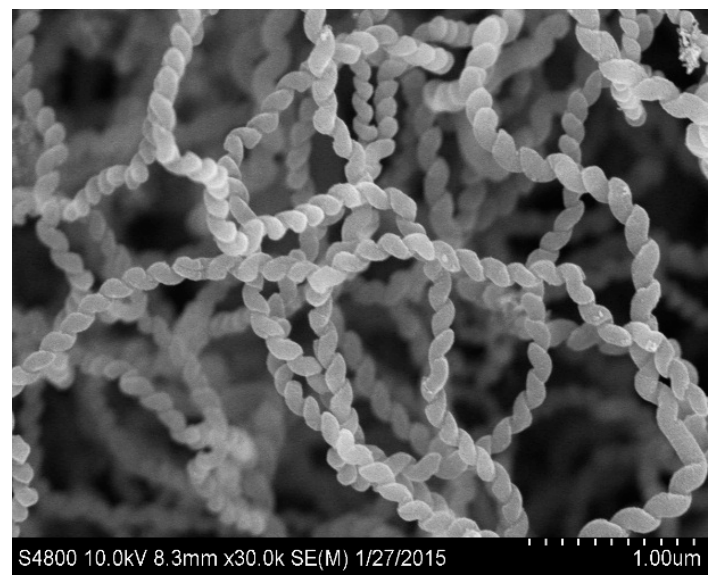

(b)

Figure 6. SEM micrographs of carbon-nanocoils at (a) low magnification and (b) high magnification. 
A TEM image of the CNCs synthesized at $600{ }^{\circ} \mathrm{C}$ is shown in the inset of Figure 7. The CNCs synthesized at this temperature had a diameter of around $100 \mathrm{~nm}$ and an amorphous structure. A Pd catalyst particle was observed in the micrograph (the red circle). Also shown in Figure 7 is the energy dispersive X-ray (EDX) spectrum that was obtained within the red circle to reveal the chemical composition of the catalyst particle (The $\mathrm{Cu}$ signal came from the $\mathrm{Cu}$ grid supporting the $\mathrm{CNC}$ samples for TEM analysis).

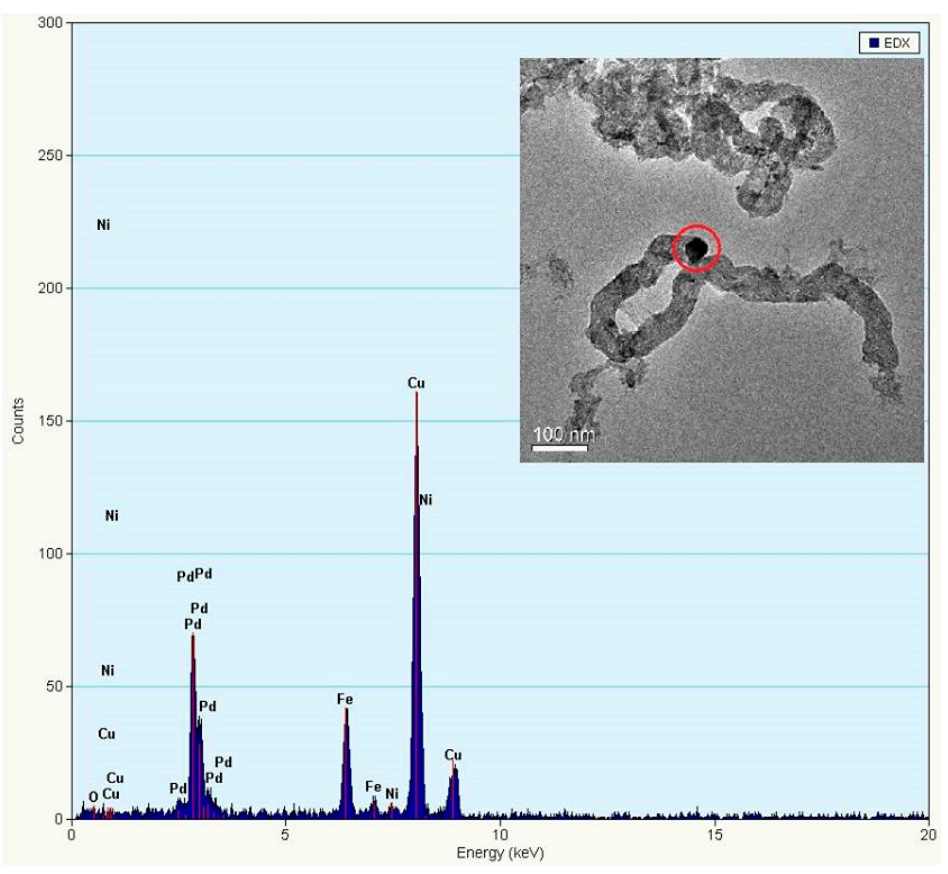

Figure 7. TEM image of CNCs (inset) and EDX spectrum taken within the red circle, which contained one Pd catalyst particle.

Figure 8 shows the Raman spectrum of the synthesized CNCs. The D-band at around $1350 \mathrm{~cm}^{-1}$ and the G-band at $1580 \mathrm{~cm}^{-1}$ are clearly seen; they are related to the structural defects and the graphite lattice, respectively, in carbonaceous materials. The intensity ratio $I_{\mathrm{D}} / I_{\mathrm{G}}$, which is an indicator of the crystallinity of carbon materials, is 0.78 . This number is similar to those obtained for the CNCs in previous studies $[39,42]$.

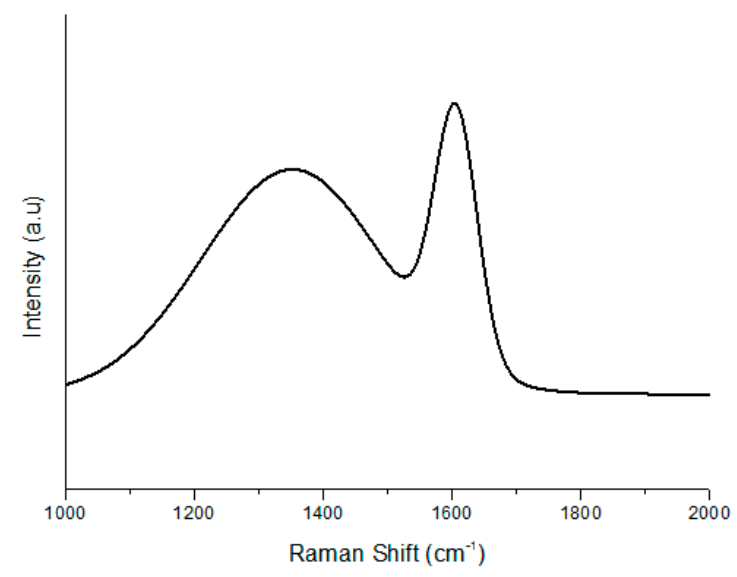

Figure 8. Raman spectrum of as-grown CNC film. 


\subsection{Test of X-Ray Tube}

To validate the device simulation herein, an X-ray tube with the designated geometry and dimensions (a $20 \mathrm{~mm}$ cathode-anode separation; a $15 \mathrm{~mm}$ diameter; a $5 \mathrm{~mm}$ focusing cup height; and a $\mathrm{Cu}$ anode) was constructed and tested. The focal spot size was estimated experimentally to be roughly $\leq 0.8 \mathrm{~mm}$. The deviation from the optimal simulation result might be attributed to the slightly different electric field distribution in the real device with the tilted anode, as shown in Figure 9, or to the non-ideal alignment of the anode relative to the cathode. The most important applications of X-rays are the nondestructive inspection of electronic devices and medical diagnosis. To demonstrate the good resolution of the cathode design herein, X-ray images of a fine test circuit board and a human tooth (Figure 10) were obtained using our X-ray tube at $50 \mathrm{kV}$. The FE current was $0.076 \mathrm{~mA}$ at $50 \mathrm{kV}$. The image was taken using a film placed behind the objects, which were irradiated by the X-ray tube. The sample to be analyzed was placed in the direction perpendicular to the cathode-anode axis (i.e., to the right side of the anode in Figure 9). The source-to-sample and sample-to-film distances were 10 and $20 \mathrm{~cm}$, respectively. The film used for the imaging was Kodak Dental Intraoral D-Speed (Kodak, Rochester, NY, USA), and the exposure time was determined by adjusting the mAs for a gross optical density of about 1.5 on a portion of the film that was not covered by the test pattern. As shown in Figure 10, the fine structures of the test circuit board and the tooth were clearly resolved using our $\mathrm{X}$-ray tube. The image quality is likely to be similar to that achieved by the conventional X-ray imaging technique, as judged by the fine details in the image of the reference phantom.

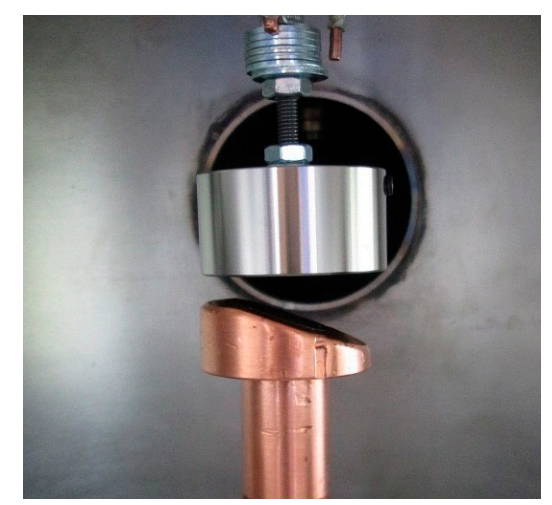

Figure 9. Photograph of the anode and cathode geometry in our X-ray tube; the anode material is $\mathrm{Cu}$.

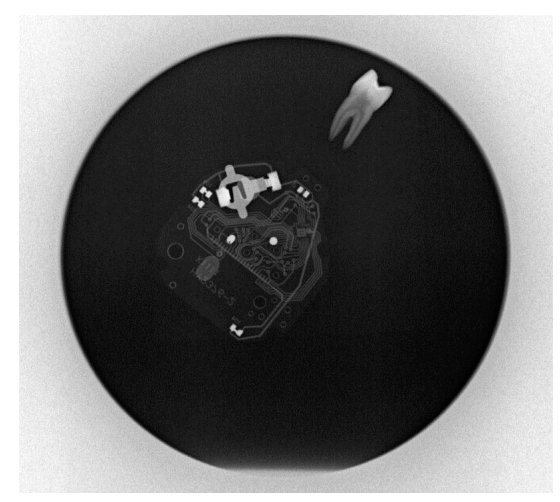

Figure 10. X-ray image of a circuit board and a tooth captured using the self-focusing X-ray tube. 


\section{Conclusions}

A self-focusing cathode with an auxiliary metallic cup was developed for use in an X-ray tube. The design was both simulated and fabricated. CNCs were chosen as electron emitters because they have a higher long-term stability, longer lifetime, higher FE currents, and more emission sites than CNTs. A disk-shaped stainless steel substrate was deposited with CNCs and placed at the bottom of the cup as the electron source. A planar copper anode was also used. The diameters for the anode and the CNC FE disk were both $15 \mathrm{~mm}$, and the aperture on the top of the cup had a diameter of $12 \mathrm{~mm}$. The primary advantages of the cup-shaped cathode were the large emitting area, the self-focusing of emitting electrons, the simplicity of the structure and the bias circuit, and the ease of fabrication. The simulation results revealed that at a cup height of $5 \mathrm{~mm}$, the optimum focal spot size was about $0.1 \mathrm{~mm}$. An X-ray tube with the designed geometry and dimensions was constructed and tested. The experimental results verified the good imaging performance of this design. The good resolution of the captured image of a fine test circuit and a tooth suggested the practical usefulness of the design.

\section{Acknowledgments}

This work was sponsored by the Ministry of Science and Technology of Taiwan under Grants MOST 103-2221-E-606-003 and MOST 104-2623-E-155-007-ET.

\section{Author Contributions}

Yih-Ming Liu and Ming-Der Ger conceived and designed the experiments; Kevin Cheng performed the experiments; Yi-Ping Chou and Kung-Hsu Hou analyzed the data; Meng-Jey Youh contributed reagents/materials/analysis tools; Nen-Wen Pu wrote the paper.

\section{Conflicts of Interest}

The authors declare no conflict of interest.

\section{References}

1. Bushong, S.C. Radiologic Science for Technologist: Physics, Biology, and Protection; Mosby: St. Louis, MO, USA, 1997; p. 5.

2. Fowler, R.H.; Nordheim, L. Electron emission in intense electric fields. Proc. R. Soc. Lond. A 1928, 119, 173-181.

3. Kuznetzov, A.A.; Lee, S.B.; Zhang, M.; Baughman, R.H.; Zakhidov, A.A. Electron field emission from transparent multiwalled carbon nanotube sheets for inverted field emission displays. Carbon 2010, 48, 41-46.

4. Zhang, Y.; Deng, S.Z.; Xu, N.S.; Chen, J. Fully sealed carbon nanotube flat-panel light source and its application as thin film transistor-liquid-crystal display backlight. J. Vac. Sci. Technol. B 2008, 26, 1033-1037.

5. Bae, N.Y.; Bae, W.M.; Ha, A.N.; Nakamoto, M.; Jang, J.; Park, K.C. Low-voltage driven carbon nanotube field emission lamp. Curr. Appl. Phys. 2011, 11, S86-S89. 
6. Wang, S.; Calderon, X.; Peng, R.; Schreiber, E.C.; Zhou, O.; Chang, S. A carbon nanotube field emission multipixel X-ray array source for microradiotherapy application. Appl. Phys. Lett. 2011, 98, 213701.

7. Whitlock, R.R.; Bell, M.I.; Kerns, D.V.; Kerns, S.; Davidson, J.L.; Kang, W.P. Transmission Cathode for X-ray Production. U.S. Patent 6,333,968, 25 December 2001.

8. Sugie, H.; Tanemura, M.; Filip, V.; Iwata, K.; Takahashi, K.; Okuyama, F. Carbon nanotubes as electron source in an X-ray tube. Appl. Phys. Lett. 2001, 78, 2578-2580.

9. Bandaru, P.R. Electrical properties and applications of carbon nanotube structures. J. Nanosci. Nanotechnol. 2007, 7, 1239-1267.

10. Zou, R.; Hu, J.; Song, Y.; Wang, N.; Chen, H.; Chen, H. Carbon nanotubes as field emitter. J. Nanosci. Nanotechnol. 2010, 10, 7876-7896.

11. Sarada, Y.; Agarwal, S.; Kar, K.K. A Simple Method for Controlled Growth of Carbon Nanocoils on Metallic Wire by Chemical Vapor Deposition. Nanosci. Nanotechnol. Lett. 2009, 1, 213-217.

12. Zhang, M.; Nakayama, Y.; Pan, L. Synthesis of carbon tubule nanocoils in high yield using iron-coated indium tin oxide as catalyst. Jpn. J. Appl. Phys. 2000, 39, 1242-1244.

13. Chen, X.; Yang, S.; Motojima, S. Morphology and growth models of circular and flat carbon coils obtained by the catalytic pyrolysis of acetylene. Mater. Lett. 2002, 57, 48-54.

14. Hernadi, K.; Fonseca, A.; Nagy, J.B.; Fudala, Á.; Bernaerts, D.; Kiricsi, I. Catalytic production of carbon nanofibers over iron carbide doped with $\mathrm{Sn}^{2+}$. Appl. Catal. A 2002, 228, 103-113.

15. Chen, X.; Saito, T.; Kusunoki, M.; Motojima, S. Three-dimensional vapor growth mechanism of carbon microcoils. J. Mater. Res. 1999, 14, 4329-4336.

16. Chen, X.; Motojima, S. The growth patterns and morphologies of carbon micro-coils produced by chemical vapor deposition. Carbon 1999, 37, 1817-1823.

17. Liu, H.; Saito, Y. Influence of surface roughness on field emission of electrons from carbon nanotube films. J. Nanosci. Nanotechnol. 2010, 10, 3983-3987.

18. Zhang, Z.; He, P.; Sun, Z.; Feng, T.; Chen, Y.; Li, H.; Tay, B. Growth and field emission property of coiled carbon nanostructure using copper as catalyst. Appl. Surf. Sci. 2010, 256, 4417-4422.

19. Sung, W.Y.; Ok, J.G.; Kim, W.J.; Lee, S.M.; Yeon, S.C.; Lee, H.Y.; Kim, Y.H. Synthesis and field emission characteristics of carbon nanocoils with a high aspect ratio supported by copper micro-tips. Nanotechnology 2010, 18, 245603.

20. Zhang, J.; Cheng, Y.; Lee, Y.Z.; Gao, B.; Qiu, Q.; Lin, W.L.; Lalush, D.; Lu, J.P.; Zhou, O. A nanotube-based field emission X-ray source for microcomputed Tomography. Rev. Sci. Instrum. 2005, 76, 094301.

21. Cheng, Y.; Zhou, O. Electron field emission from carbon nanotubes. Physique 2003, 4, 1021-1033.

22. Murphy, E.L.; Good, R.H., Jr. Thermionic Emission, Field Emission, and the Transition Region. Phys. Rev. 1956, 102, 1464-1473.

23. Dyke, W.P.; Dolan, W.W. Field emission. Adv. Electron. Electron. Phys. 1956, 8, 89-183.

24. Gadzuk, J.W.; Plummer, E.W. Field Emission Energy Distribution (FEED). Rev. Mod. Phys. 1973, 45, 487-548.

25. Gomer, R. Field emission, field ionization and field desorption. Surface Sci. 1994, 299-300, $129-152$. 
26. De Heer, W.A.; Ugarte, D. Carbon onions produced by heat treatment of carbon soot and their relation to the $217.5 \mathrm{~nm}$ interstellar absorption feature. Chem. Phys. Lett. 1993, 207, 480-486.

27. Fan, S. Self-oriented regular arrays of carbon nanotubes and their field emission properties. Science 1999, 283, 512-514.

28. Rinzler, A.G.; Hafner, J.H.; Nikolaev, P.; Nordlander, P.; Colbert, D.T.; Smalley, R.E.; Lou, L.; Kim, S.G.; Tomanek, D. Synthesis of Large Arrays of Well-Aligned Carbon Nanotubes on Glass. Science 1995, 269, 1550-1553.

29. De Heer, W.A.; Châtelain, A.; Ugarte, D. A Carbon Nanotube Field-Emission Electron Source. Science 1995, 270, 1179-1180.

30. Saito, Y.; Uemura, S. Field emission from carbon nanotubes and its application to electron sources. Carbon 2000, 38, 169-182.

31. Bonard, J.M.; Croci, M.; Klinke, C.; Kurt, R.; Noury, O.; Weiss, N. Carbon nanotube films as electron field emitters. Carbon 2002, 40, 1715-1728.

32. Huang, J.X.; Chen, J.; Deng, S.Z.; She, J.C.; Xu, N.S. Field-emission fluorescent lamp using carbon nanotubes on a wire-type cold cathode and a reflecting anode. J. Vac. Sci. Technol. B 2008, doi:10.1116/1.2970144.

33. Downs, W.B.; Baker, R.T.K. Novel carbon fiber-carbon filament structures. Carbon 1991, 29, $1173-1179$.

34. Motojima, S.; Kawaguchi, M.; Nozaki, K.; Iwanaga, H. Preparation of coiled carbon fibers by catalytic pyrolysis of acetylene, and its morphology and extension characteristics. Carbon 1991, 29, 379-385.

35. Kawaguchi, M.; Nozaki, K.; Motojima, S.; Iwanaga, H. A growth mechanism of regularly coiled carbon fibers through acetylene pyrolysis. J. Cryst. Growth 1992, 118, 309-313.

36. Chen, X.; Motojima, S. Morphologies of carbon micro-coils grown by chemical vapor deposition. J. Mater. Sci. 1999, 34, 5519-5524.

37. Chesnokov, V.V.; Zaikovskii, V.I.; Buyanov, R.A. Symmetric twisted carbon filaments formed from butadiene-1,3 on Ni-Cu/MgO-catalyst: Growth regularities and mechanism. J. Mol. Catal. A 2000, 158, 267-270.

38. Pu, N.W.; Youh, M.J.; Chung, K.J.; Liu, Y.M.; Ger, M.D. Field emission lamps prepared with dip-coated and nickel electroless plated carbon nanotube cathodes. J. Nanosci. Nanotechnol. 2015, 15, 5093-5098.

39. Chung, K.J.; Pu, N.W.; Youh, M.J.; Liu, Y.M.; Ger, M.D.; Cheng, K.; Jiang, J.C. Improvement of Field-Emission-Lamp Characteristics Using Nitrogen-Doped Carbon Nanocoils. Diam. Relat. Mater. 2015, 53, 1-10.

40. Chung, K.J.; Pu, N.W.; Youh, M.J.; Liu, Y.M.; Ger, M.D.; Huang W.K. Improvement of Lighting Uniformity and Phosphor Life in Field Emission Lamps Using Carbon Nano-Coils. J. Nanomater. 2015, in press.

41. Chou, Y.P.; Pu, N.W.; Ger, M.D.; Chung, K.J.; Hou, K.H.; Liu, Y.M. Bulb-Shaped Field Emission Lamps Using Carbon Nano-Coil Cathodes. J. Nanosci. Nanotechnol. 2012, 12, 8316-8322. 
42. Huang, W.K.; Chung, K.J.; Liu, Y.M.; Ger, M.D.; Pu, N.W.; Youh, M.J. Carbon Nanomaterials Synthesized Using a Spray Pyrolysis Method. Vacuum 2015, 118, 94-99.

(C) 2015 by the authors; licensee MDPI, Basel, Switzerland. This article is an open access article distributed under the terms and conditions of the Creative Commons Attribution license (http://creativecommons.org/licenses/by/4.0/). 